\title{
The outcomes of thermal symmetry after orofacial pain acupuncture treatment
}

\author{
by R. Vardasca*** , M. Clemente* ${ }^{*}$ A. Pinto******* and J. Gabriel* \\ * LABIOMEP, UISPA-LAETA-INEGI, Faculty of Engineering, University of Porto, Rua Dr. Roberto Frias 404, \\ 4200-465 Porto, Portugal, rvardasca@fe.up.pt \\ ** Medical Imaging Research Unit, Faculty of Computing, Engineering and Science, University of South Wales, \\ Pontypridd, CF37 1DL, United Kingdom \\ ${ }^{* * \star}$ Centro Hospitalar do Porto EPE, Largo Prof. Abel Salazar, 4099-001 Porto, Portugal \\ **** Instituto de Ciências Biomédicas Abel Salazar da University of Porto, Rua de Jorge Viterbo Ferreira 228, \\ 4050-313 Porto, Portugal
}

\begin{abstract}
Orofacial pain is a common health condition, which affects considerably the life quality of patients. Some of these patients are sensitive to treatment drugs and have to look for alternative treatments. Acupuncture seems to be an adequate treatment in this situation, however like most treatments its evaluation relies in the subjectivity of the patient claims. A methodology using infrared imaging in frontal and lateral views of the face, before and after treatments was performed. In the obtained images regions of interest were drawn and measured and the value of thermal symmetry used to quantitatively evaluate and prove the treatment effect.
\end{abstract}

\section{Introduction}

Orofacial pain has been defined as pain localized to the region above the neck, in front of the ears and below the orbitomeatal line, as well as pain within the oral cavity, which can include pain of dental origin and temporomandibular disorders [1]. However, some orofacial pain conditions may involve areas outside this region. This kind of pain has physiological manifestations, namely at the microvascular and autonomic nervous systems, which affect the thermal imbalance of the skin, infrared imaging can be used to assess this changes [2] and the thermal symmetry value calculated to assess its stage and treatments outcome [3]. Acupuncture has been suggested as treatment to patients that present sensitivity to the use of drugs [4].

\section{Methodology}

Seven patients of the orofacial pain service at the Centro Hospital do Porto were selected and screened with infrared imaging, following the international capture guidelines [5-7]. The views taken were the frontal and lateral face. Regions of interest were drawn using the facial location of acupuncture points (Fig. 1) and the value of thermal symmetry was calculated [3].

\section{Results}

Examples of images taken are presented in Fig. 2 and example of one patient evaluation charts based in regions of interest thermal symmetry values are shown in Fig. 3, where can be easily seen that after treatments this value decreased correlating the subjective claims of the patients, that the pain was also decreasing.

\section{Conclusions}

The developed methodology using infrared imaging to evaluated alternative treatments to drug sensitive orofacial pain patients was objective and repeatable, being an important addition to physicians that engage in this practice to monitor the treatment progress and demonstrate it to the patients, documenting their health status objectively.

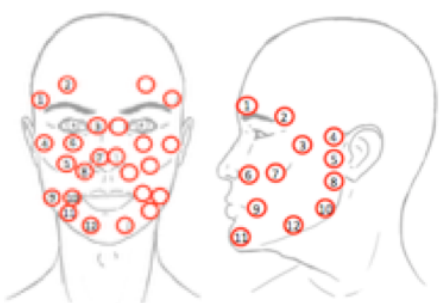

Fig. 1. Regions of interest based in the facial acupuncture points, frontal (left), lateral (right). 

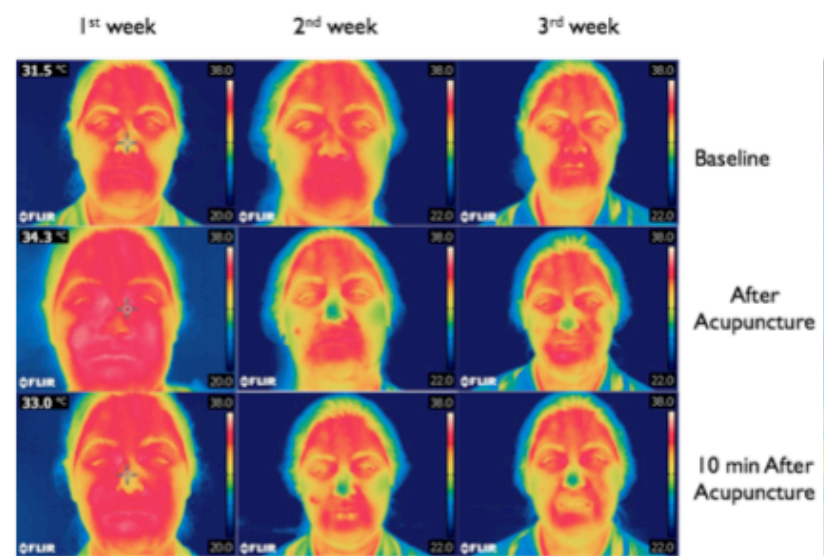

Ist week

$2^{\text {nd }}$ week

$3^{\text {rd }}$ week

Fig. 2. - Examples of facial infrared images taken, frontal (left) and lateral (right), during three treatments.
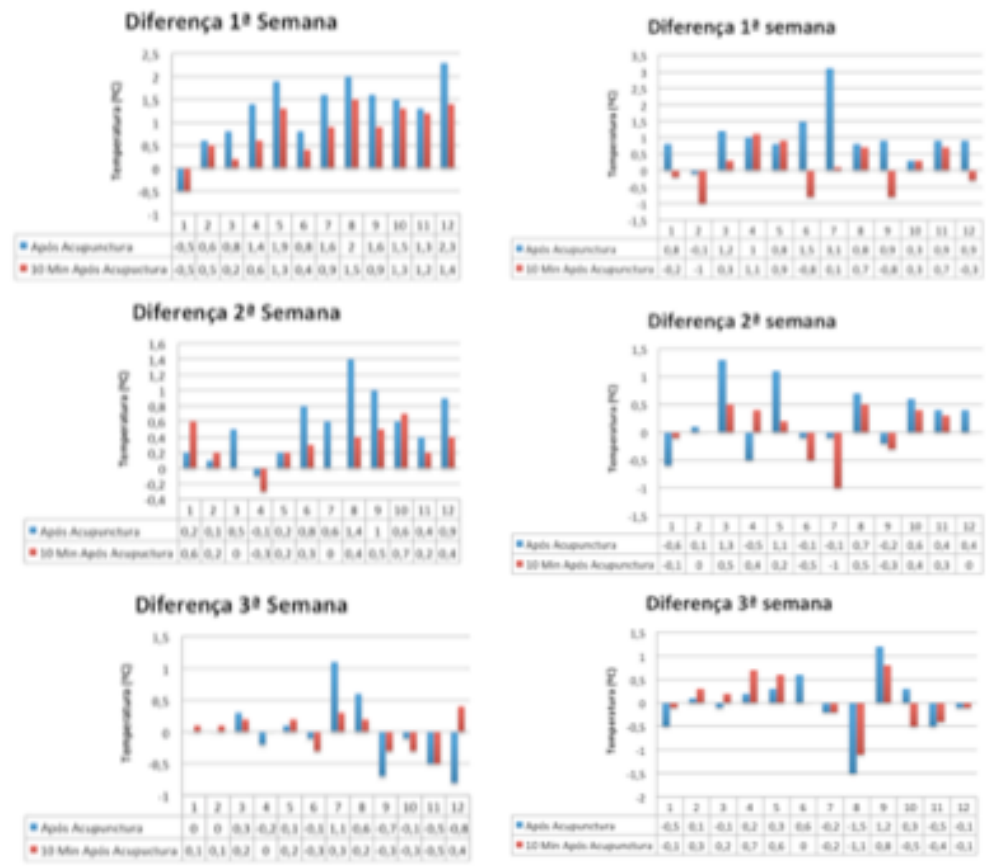

Fig. 3. - Examples of thermal assessment of views, frontal (left) and lateral (right), during three treatments.

\section{REFERENCES}

[1] Shephard M. K., MacGregor E. A., Zakrzewska J. M. "Orofacial pain: a guide for the headache physician". Headache: The Journal of Head and Face Pain, 54(1), 22-39, 2014.

[2] Ring E. F. J., Ammer K. "Infrared thermal imaging in medicine". Physiological measurement, 33(3), R33-R46, 2012.

[3] Vardasca R., Ring E. F. J., Plassmann P., Jones C. D. "Thermal Symmetry of the limbs in healthy subjects". Thermology International, 22(2), 53-60, 2012.

[4] Goddard G., Karibe H., McNeill C., Villafuerte, E. "Acupuncture and sham acupuncture reduce muscle pain in myofascial pain patients". Journal of orofacial pain, 16(1), 71-76, 2001.

[5] Ring E. F. J., Ammer K. "The technique of infrared imaging in medicine". Thermology international, 10(1), 7-14, 2000.

[6] Schwartz R. G. "Guidelines For Neuromusculoskeletal Thermography”. Thermology international, 16(1), 5-9, 2006.

[7] Ammer K. "The Glamorgan Protocol for recording and evaluation of thermal images of the human body". Thermology international, 18(4), 125-144, 2008. 